\section{Wandering spleen: a rare clinical entity}

\section{Introduction}

Wandering, ectopic, splenoptosis spleen is a mobile spleen without peritoneal attachments. It is attached with an elongated vascular pedicle which permits it to move to any part of the abdomen or pelvis. ${ }^{1}$ Wandering spleen is uncommon and results due to congenital mal-development or due to acquired laxity of peritoneal attachments including the lienorenal and gastrosplenic ligaments. ${ }^{2}$ Ectopic, migrating or splenoptosis are synonymous with wandering spleen. Spleen can move to the lower abdomen or pelvis. ${ }^{3}$ It is more commonly encountered in women of reproductive age, and may be misdiagnosed as an abdominal or adenxal mass. ${ }^{3}$ Ultrasound imaging is least invasive and more effective for diagnosis. Early diagnosis and surgical treatment is mandatory to prevent complications. The first case of wandering spleen was reported by Hatfield et al in $1976 .{ }^{4}$ We present a 40 -year-old woman with a painful abdominal lump. Her ultrasound showed an enlarged ectopic hypoechoic mass in the right iliac fossa with a large vascular pedicle and was treated by laparotomy.

\section{Case report}

A 40-year-old woman presented with a six month history of dull aching pain and lump in the right iliac fossa which extended into the right half of pelvis. There was no history of any pressure symptoms such as bladder or bowel disturbances. Clinical examination revealed a $10 \times 12 \mathrm{~cm}$ intra-abdominal, non-tender, smooth mass, which was freely mobile and positioned in an oblong fashion in the right iliac fossa, extending into the pelvis. On hematological examination, the patient had hemoglobin of $8.6 \mathrm{gm} \%$, and her TLC was $5300 / \mathrm{mm}^{3}$.

Ultrasonography revealed a homogenous lobulated mass with a moderate fine echo pattern in the right iliac fossa, extending to the pelvis. A large blood vessel was seen entering the mass. The right ovary could not be detected and a differential diagnosis of solid ovarian tumor or ectopic spleen was proposed (Figure 1). After infusing three units of blood to raise her hemoglobin to $10.2 \mathrm{gm} \%$, a laparotomy was carried out through a midline incision and an enlarged spleen hanging with a long pedicle was found (Figure 2). Splenectomy was

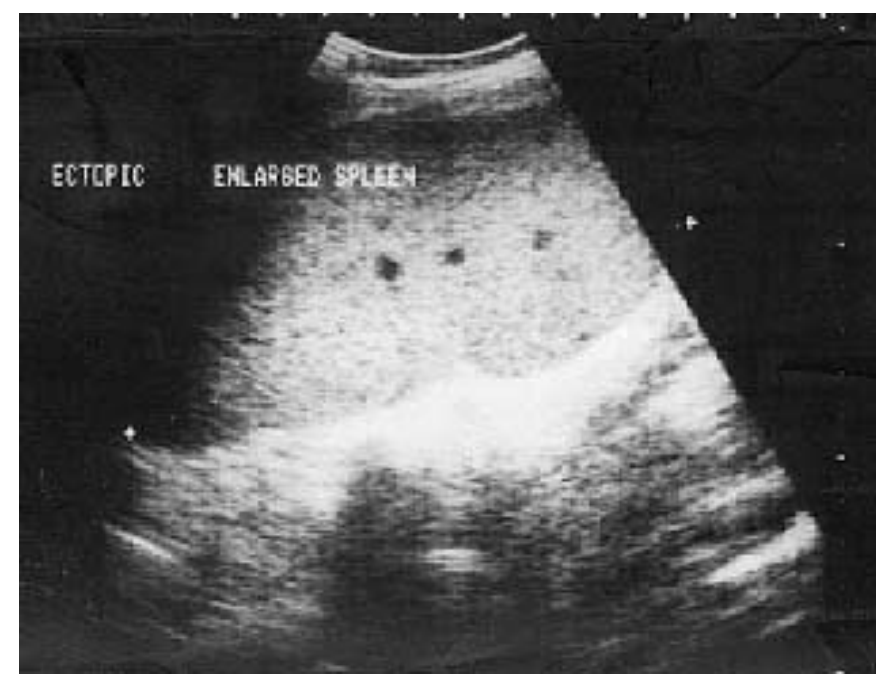

Figure 1: Ultrasound abdomen showing a large uniformly echogenic mass in the right iliac fossa.

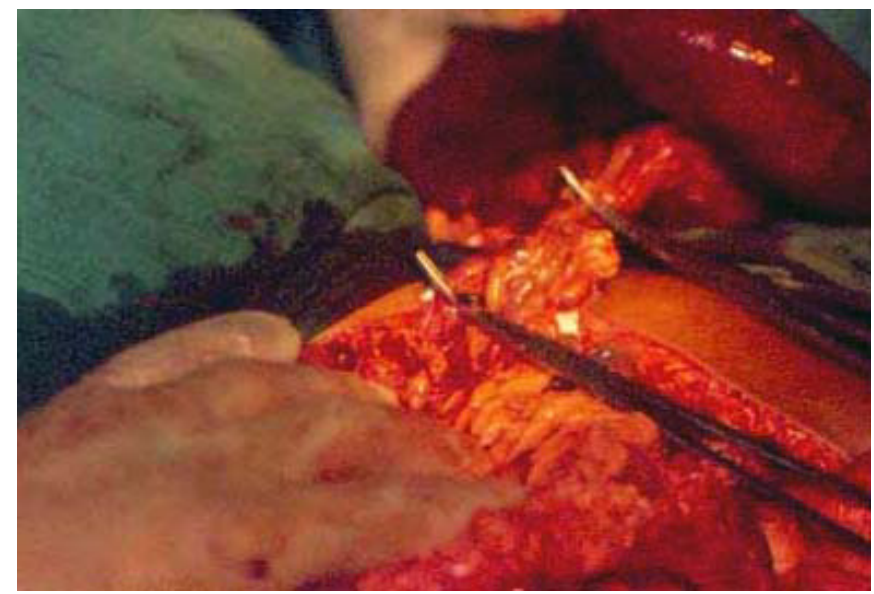

Figure 2: Laparotomy showing an enlarged spleen hanging by an elongated vascular pedicle.

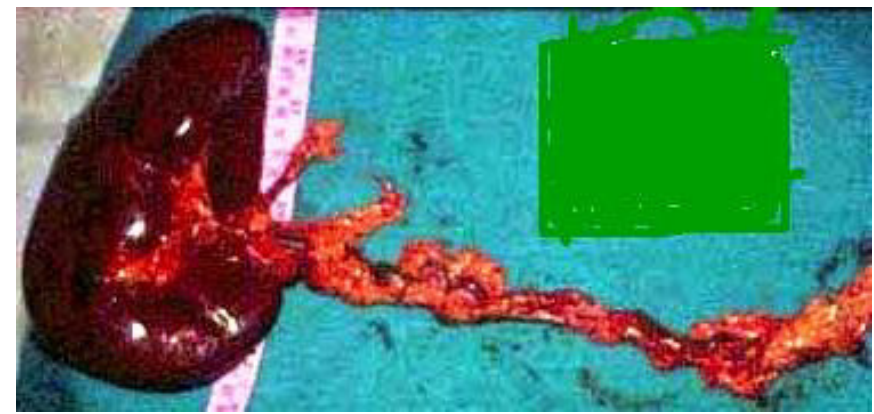

Figure 3: Gross specimen showing a huge spleen and an elongated vascular pedicle

performed (Figure 3). The postoperative period was uneventful. The patient was instituted on antibiotic prophylaxis.

\section{Discussion}

Wandering spleen refers to an ectopic location of the spleen 
within the abdomen or pelvis. The exact incidence is unknown because most of these patients are asymptomatic. Its incidence is less that $0.5 \%$ of all splenectomies. ${ }^{5}$ It affects both sexes but is more common in women of reproductive age and in children. About 500 patients with wandering pelvic spleen have been reported in the literatures so far. ${ }^{4}$

A wandering spleen may be an incidental finding on physical or radiographic examination. Clinical diagnosis may be quite difficult. Some authors suggest that the diagnosis can be made with certainty if the following criteria are met: (1) palpation of a mass with a notched edge; (2) mobility of the mass, which is painless in the left upper quadrant and painful in other directions; (3) resonant on percussion in left upper quadrant; and (4) chronic intermittent pain. ${ }^{6}$

The definitive treatment of a wandering spleen is surgery since non-operative treatment is associated with a high rate of complications (as high as 65\%). Surgery includes splenopexy for selected asymptomatic patients. It preserves splenic functions and avoids the potential danger of post-splenectomy sepsis which is seen in $1.9 \%$ adults and $4 \%$ children. Given its physiologic importance especially in children and the risk of post-splenectomy sepsis, an early diagnosis and splenopexy are recommended.?

SANJEEV SINGLA ${ }^{1}$ MAMTA SINGLA ${ }^{2}$ SUNDEEP SINGLA ${ }^{3}$ SUNDER GOYAL ${ }^{1}$

Correspondence: Dr. Sunder Goyal Department of General and Minimal Invasive Surgery, ${ }^{I}$ BPS Government Medical College for Women, Khanpur kalan, Sonepat, Haryana, India Departments of Surgery ${ }^{2}$ and Medicine, ${ }^{3}$ Rama Medical College and Hospital, Ghaziabad, India Email:goyal.sunder@yahoo.in

\section{References}

1. Bouassida M, Sassi S, Chtourou MF, Bennani N, Baccari S, Chebbi F, et al. A wandering spleen presenting as a hypogastric mass: case report. Pan Afr Med J. 2012;11:31.

2. DeJohn L, Hal H, Winner L. Wandering spleen: a rare diagnosis with variable presentation. Radiology Case Reports. [Online] 2008;3:229.

3. Chan KC, Chang YH. Acute abdomen due to torsion of a pelvic wandering spleen. J Formos Med Assoc. 2002;101:577-80.

4. Zandi B, Zandi N. Wandering spleen presenting as an asymptomatic pelvic mass: a case report. Iran J Radiol.
2008;5:141-4.

5. Sayeed S, Koniaris LG, Kovach SJ, Hirokawa T. Torsion of a wandering spleen. Surgery. 2002;132:535-6.

6. Balik E, Yazici M, Taneli C, Ulman I, Genc K. Splenoptosis (wandering spleen). Eur J Pediatr Surg. 1993;3:174-5.

7. Soleimani M, Mehrabi A, Kashfi A, Fonouni H, Buchler MW, Kraus TW. Surgical treatment of patients with wandering spleen: report of six cases with a review of the literature. Surg Today. 2007;37:261-9.

\section{Anal symptoms to anal obliteration: quackery in proctology continues!}

\section{Introduction}

The scarcity of trained medical professionals continues to be a major concern in developing countries. Lack of stringent regulations permits quacks to impersonate as physicians and administer unscientific and potentially harmful treatment to gullible patients. Even in the $21^{\text {st }}$ century, we continue to come across anorectal complications arising from unscientific management of haemorrhoids by quacks. Quacks are known to inject corrosive preparations in anal fistula as remedy. However such quackery often leads to various local complications including necrotizing fasciitis of the perineum and scrotum, and systemic complications like septicemia and renal failure due to systemic absorption of chemical agents. ${ }^{1}$ We report a case of complete anal obliteration following application of a corrosive preparation into the anus by a quack doctor for hemorrhoids, and describe its successful management at our hospital.

\section{Case report}

A 25-year-old lady presented with pain, distension of abdomen, and constipation of seven days duration. One month ago, the lady had applied a corrosive preparation into the anus on advice of a quack doctor, purportedly for the treatment of hemorrhoids. Soon after, she felt intense pain which subsided gradually; however, progressive constipation ensued. Abdominal examination revealed features of intestinal obstruction. A scar measuring $6.5 \times 2.5 \mathrm{~cm}$ was found at the anus and the anus 